\title{
Seemingly intersecting flaring loops in microwaves: Possible radio evidence for reconnection
}

\author{
M. R. Kundu ${ }^{1,2}$ and V. V. Grechnev ${ }^{3,4}$ \\ ${ }^{1}$ National Astronomical Observatory, Mitaka, Tokyo 181, Japan \\ ${ }^{2}$ University of Maryland, College Park, MD 20742, U.S.A. \\ ${ }^{3}$ Nobeyama Radio Observatory, Nobeyama, Japan \\ ${ }^{4}$ Institute of Solar-Terrestrial Physics, Irkutsk, Russia
}

(Received May 18, 2000; Revised August 1, 2000; Accepted September 7, 2000)

\begin{abstract}
We present possible radio evidence of magnetic reconnection in a solar flare — an LDE event of GOES class M3.2 that was observed on 1999 February 16 on the disk. Our evidence is based upon the facts that we observed a bright microwave source-blob at 17 and $34 \mathrm{GHz}$ near the intersection of two seemingly intersecting flaring loops or as a loop-top source above one flaring loop. The bright microwave source is clearly non-thermal and its time profile is similar to those of the flaring footpoint microwave sources; and in the late decay phase of the event the microwave source structure is similar to that of the corresponding SXR source.
\end{abstract}

\section{Introduction}

Magnetic reconnection has always played a key role in solar flare models since the early days (Parker, 1957; Sweet, 1958; Petschek, 1964). Specifically it has been proposed that magnetic reconnection in current sheets is the primary energy source in solar flares. However, providing direct evidence of reconnecting current sheets is not easy and most observations in the past in any spectral domain have been necessarily interpretative. The role of magnetic reconnection in solar flares received a well-deserved revival over the past several years from Yohkoh/SXT and HXT observations. In particular, the works of Tsuneta et al. (1992) and Tsuneta (1996) on the observational side and the theoretical studies by Shibata (1996) and Shibata et al. (1995) are noteworthy. According to them, magnetic reconnection at a neutral sheet located at the top of a flaring loop is responsible for energy release in at least some flares. The initial analysis by Tsuneta et al. (1992) of an LDE flare that occurred on 1992 February 21 led them to believe that magnetic reconnection occurs around the top of the flaring loop. For their conclusions they depended upon several crucial evidence: 1) The loop-top region has a cusp-shaped structure, implying the location of a reconnection site at the top of the flaring loop. 2) The height of soft X-ray flaring loop and foot point separation increase with increasing time, with a speed of order $10 \mathrm{~km} \mathrm{sec}^{-1}$. They interpreted this rise speed as due to the rise of the X-point location along the neutral sheet as reconnection progresses. 3) They also provided further evidence that outer flaring loops have higher temperatures in the decay phase because of being reconnected later than the inner flaring loop—consistent with flare energy being supplied from reconnection process near the top of the flaring loop. Further indirect evidence of

Copy right $(\mathrm{C}$ The Society of Geomagnetism and Earth, Planetary and Space Sciences (SGEPSS); The Seismological Society of Japan; The Volcanological Society of Japan; The Geodetic Society of Japan; The Japanese Society for Planetary Sciences. magnetic reconnection in current sheets is provided by spectral line observations in the soft X-ray (SXR) domain of fast plasma outflows with velocities up to about $200 \mathrm{~km} \mathrm{sec}^{-1}$ (Antonucci et al., 1996; McKenzie and Hudson, 1999). In the hard X-ray (HXR) domain, using the HXT imaging data, Masuda et al. (1994) discovered that in some impulsive limb flares, a loop-top HXR source appeared well above the SXR bright loop during the impulsive phase. This was interpreted by Shibata et al. (1995) as resulting from magnetic reconnection occurring above the loop-a high-speed jet is created through magnetic reconnection, which collides with the loop top, producing a fast shock, super-hot plasma and/or highenergy electrons emitting HXR. This example illustrates that magnetic reconnection could occur during the impulsive phase as well as in the decay phase of a long duration event (LDE) flares.

In the radio domain, providing evidence of magnetic reconnection, however indirect, is extremely difficult because of relatively poor spatial resolution of radio telescopes compared with X-ray telescopes. Further in the radio domain, it is not possible to measure velocities of plasma flows by means of spectral lines; one has to depend upon geometri$\mathrm{cal} /$ morphological features of flaring structures. However, in the microwave radio domain reliable measurements of magnetic polarities are possible, and thus flaring loops can be identified rather easily (especially when there is polarized footpoint emission). In the past, Kundu et al. (1982) and Kundu (1985) reported $6 \mathrm{~cm}(5 \mathrm{GHz})$ observations of a flare using the VLA with a spatial resolution of $2^{\prime \prime}$; they presented total intensity $(I)$ and polarization $(V)$ maps which could be interpreted only as due to possible magnetic reconnection resulting from interaction of an emerging flux region with the overlying magnetic structure. In particular they showed evidence of a quadrupole structure (two oppositely polarized twisted flux tubes in close proximity to each other) at the 
peak of an impulsive flare, suggestive of such a structure in a specific flare model (Gold and Hoyle, 1960). However, one has to recognize that the VLA is not a dedicated solar radio telescope, and no matter how good the spatial resolution is, one has to be fortunate to come across flaring structures, suggestive of reconnection process. By contrast, the Nobeyama Radio Heliograph (NoRH) is a dedicated microwave telescope, operating at 17 and $34 \mathrm{GHz}$, with which it is possible to search more efficiently for microwave signatures of magnetic reconnection in solar flares. Nevertheless, there are some constraints that one has to remember, namely that the spatial resolution of NoRH is $\gtrsim 10^{\prime \prime}$ at $17 \mathrm{GHz}$ and $\gtrsim 5^{\prime \prime}$ at $34 \mathrm{GHz}$, and so the flaring structures have to be relatively large so that one can detect microwave signatures of magnetic reconnection in a flare. In this paper we present possible microwave signature of magnetic reconnection in a solar flare. The flare in question was observed on 1999 February 16; it was an LDE flare. Note that many LDE flares have been discussed in the X-ray domain in the context of magnetic reconnection.

\section{Observations}

A long duration event (LDE) of GOES class M 3.2 that occurred on 1999 February 16 in active region NOAA 8458 was observed by the NoRH at 17 and $34 \mathrm{GHz}$ as well as by the Nobeyama polarimeters at 1, 2, 3.75, 9.4, 17 and $35 \mathrm{GHz}$. It was a disk event, located at S25 W17. According to GOES soft X-ray data, the event started at $\sim 02: 45$ UT and has a total duration of more than three hours. The initial part of the event, including the main impulsive peak, was not observed by Yohkoh/SXT and HXT, because of Yohkoh night time. However, after it came back from night, Yohkoh observed the event during the late decay phase from 03:19 UT. CGRO/BATSE has recorded HXR emission of almost the entire flare except for the period 02:55 to 02:57 UT.

Figures 1(a) and 1(b) show the GOES and BATSE time profiles. Figure 1(a) shows the GOES time profiles in two channels as well as the temperature and emission measure time profiles computed from GOES X-ray parameters. Figure 1(b) shows the CGRO/BATSE time profiles during the period 02:52-03:17 UT. It shows a small peak at $\sim 02: 52: 40$ UT in the $25-100 \mathrm{keV}$ channels, a large peak at $\sim 02: 54$ UT in the 25-100 keV range (a smaller one in the 100-300 keV range), a second larger and sharper peak at $\sim 02: 58 \mathrm{UT}$, followed by a smaller peak at $\sim 03: 05$ UT in the decay phase.

The event was observed by NoRH at 17 and $34 \mathrm{GHz}$ from about 02:50 UT. We produced images at both frequencies during the main impulsive phase, other smaller peaks, and during the decay phase at the rate of one map every $20 \mathrm{~s}$ from 02:50 to 04:16 UT. In addition, we have mapped the fine time structures in the initial phase from 02:52:00 to 02:55:00 every $5 \mathrm{~s}$. The time profiles of the event in both total intensity $(I)$ and polarization $(V)$ at $17 \mathrm{GHz}$, and in $I$ only at $34 \mathrm{GHz}$ (polarization is not measured by NoRH at $34 \mathrm{GHz}$ ) are shown in Fig. 2. Also shown in Fig. 2 are the correlation plots which sample small spatial scales $\left(<24^{\prime \prime}\right.$ at zenith) by using combined responses from the longest baselines of the radio interferometer at 17 and $34 \mathrm{GHz}$ as well as the total flux at 17 and $34 \mathrm{GHz}$ of a small area $\left(\sim 10^{\prime \prime} \times 10^{\prime \prime}\right)$ at the apparent intersection of two flaring loops (the center of this area is

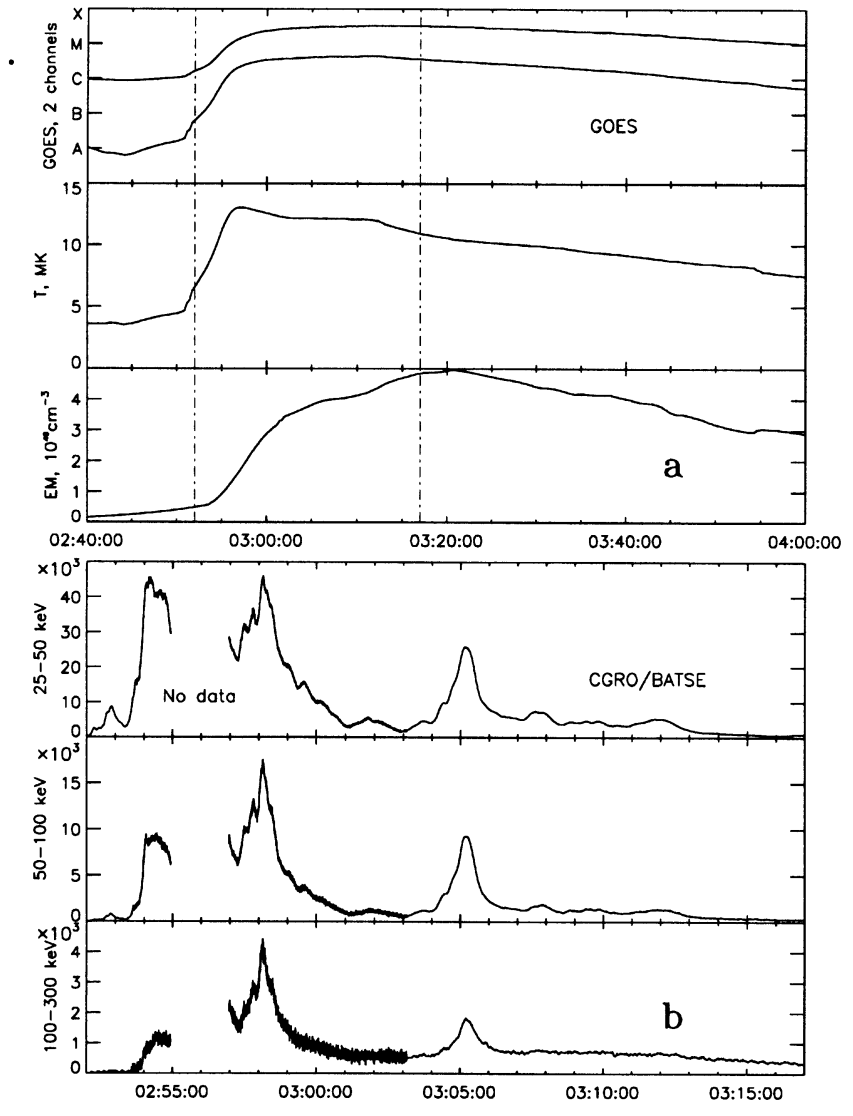

Fig. 1. M 3.2 X-ray flare of 1999 February 16 recorded by GOES (a) and CGRO/BATSE (b). a) Two GOES channels (top), temperature (middle) and emission measure (bottom) derived from GOES data. Vertical dash-dotted lines show the interval when BATSE records are available. b) Time profiles of the flare recorded by three BATSE channels and averaged over 5 points. The highest energy channel did not show adequate response. Counts should be multiplied by 1000 .

indicated by a cross in the 03:05:21 UT image of Fig. 3).

Figure 2 shows the main impulsive phase at both $17 \mathrm{GHz}$ ( $I$ and $V$ ) and $34 \mathrm{GHz}(I)$. The main phase lasts about $11 \mathrm{~min}$ (02:54-03:05), with rise phase lasting about $4.5 \mathrm{~min}$ to reach the peak at $\sim 02: 58: 30 \mathrm{UT}$, followed by a similarly rapid decay phase (from 02:58:30 to 03:05 UT). At both 17 and $34 \mathrm{GHz}$ the high-cadence correlation plots show more fine structures than the computed profiles. Note also that the smaller peak at 03:05 UT does not show up in the fourth panel (intersection region) which is important in the sense that this region may not produce the highest energy electrons emitting in the energy range $100-300 \mathrm{keV}$ at 03:05 UT, as shown in the BATSE time profiles (Fig. 1(b)). They seem to be produced at a footpoint (see below). Both the impulsive peak at $\sim 02: 58$ UT and the smaller peak at about 03:05:30 UT are moderately ( $\sim 16 \%$ ) polarized. The correlation plot at $17 \mathrm{GHz}$ shows spiky structures between 02:52:30 and 02:54:00 UT, which are suggestive of non-thermal processes. These spiky structures also appear in the $17 \mathrm{GHz}$ time profile of the intersection region (see Fig. 2).

According to the total flux time profiles recorded by Nobeyama polarimeters, the turnover frequency for the main peak is about $10 \mathrm{GHz}$ with the photon spectral index -1.5 at the high-frequency part of the spectrum, and for the smaller 


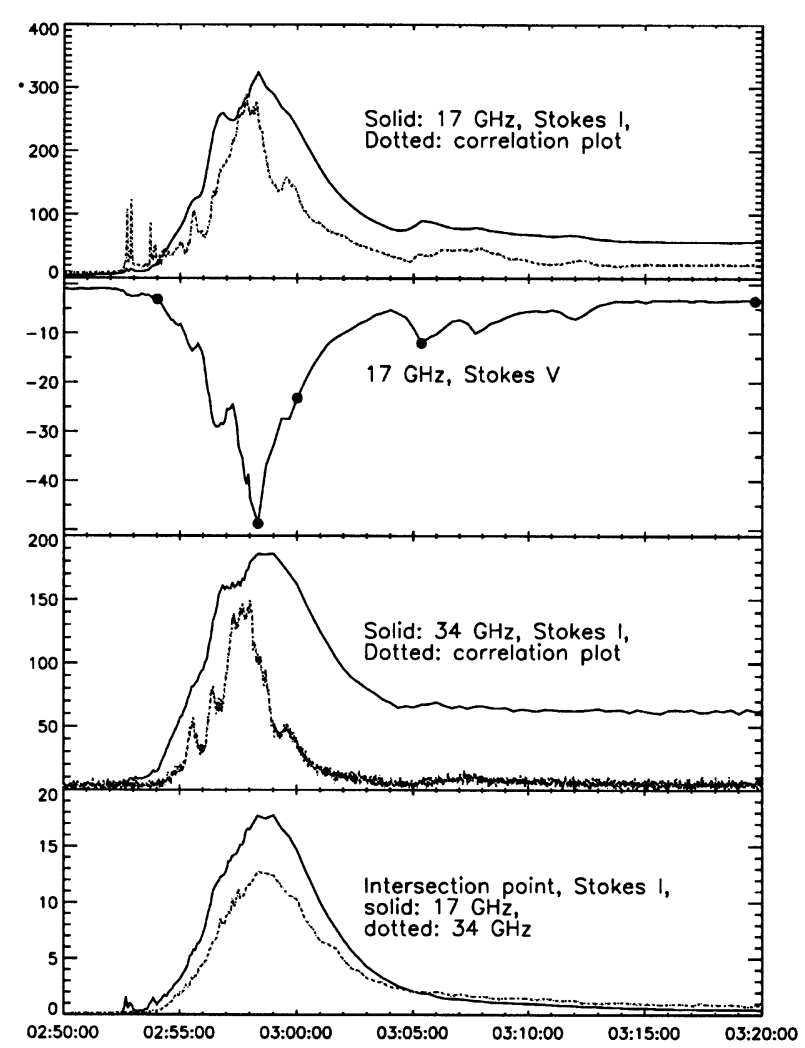

Fig. 2. The time profiles of the 1999 February 16 burst at 17 and $34 \mathrm{GHz}$ obtained with the NoRH. The first and second panels show the total intensity (Stokes $I$ ) and polarization (Stokes $V$ ) at $17 \mathrm{GHz}$, the third panel shows Stokes $I$ at $34 \mathrm{GHz}$. The fourth panel shows the Stokes $I$ of the intersection point as indicated by a cross in the 03:05:21 UT $17 \mathrm{GHz}$ image of Fig. 3. The solid curves in the first three panels are computed from NoRH images, the dotted curves in first and third panels are the correlation plots, and in the fourth panel the computed Stokes $I$ at 17 and $34 \mathrm{GHz}$ are shown by solid and dotted curves respectively. The filled circles in the second panel denote the times at which the images in Fig. 3 are shown, except the one at 03:19 UT. This time is noted because we compare the $17 \mathrm{GHz}$ and SXT images at this time (see Fig. 5).

peak at 03:05 UT they are $\sim 3 \mathrm{GHz}$ and -2.4 respectively.

Figure 3 shows four 1 -s images ( $I$ and $V$ at $17 \mathrm{GHz}$ and $I$ at $34 \mathrm{GHz}$ ) at selected times (indicated by filled circles in the $17 \mathrm{GHz} V$-plot of Fig. 2) during the rise phase, near the main peak, at the decline of the impulsive phase of the event at both 17 and $34 \mathrm{GHz}$, and at the smaller peak at 03:05:21 UT, which is superposed on the decay phase of the event. In Fig. 3 we also put contours of polarization at both $17 \mathrm{GHz} I$ and $V$-maps.

Figure 4(a) shows $17 \mathrm{GHz}$ Stokes $V$ image (averaged over the impulsive phase from 02:55 to 03:05 UT) and overlaid by contours of $17 \mathrm{GHz} I$-image (averaged over the same interval) of two apparently intersecting flaring loops on which we have marked by numbers 1, 2, 3, 4 different positions in the flaring structures. Positions 3 and 4 seem to correspond to footpoints of one flaring loop, while the second loop has one footpoint close to pos. 2, and its other positively polarized footpoint is rooted slightly southwestward from the intersection region (marked as pos. 1). The time profiles of these positions are shown in Figs. 4(b)-(e). Figure 4(b) shows the $17 \mathrm{GHz}(I, V)$ and $34 \mathrm{GHz}(I)$ time profiles of pos. 1 corresponding to the intersection region. This figure should be compared to Fig. 2-fourth panel. Figure 4(c) shows similar time profiles of pos. 2 (footpoint of one flaring loop). Note that this profile is much narrower than that of Fig. 4(b), although both peak at the same time, $\sim 02: 58: 30$ UT. That is, the footpoint emission has much faster rise $(1.5 \mathrm{~min})$ and decay ( $2 \mathrm{~min}$ ) times. Pos. 2 time profiles (in both $I, V$ ) at $17 \mathrm{GHz}$ have also a peak at 03:04-03:10 UT which overlaps the secondary BATSE peak at 03:05 UT in the energy range 25-300 keV. This peak does not exist at $34 \mathrm{GHz}$. Figure 4(d) shows similar time profiles of pos. 3 , which corresponds to a footpoint of the second flaring loop. This has a peak at 03:00 UT and polarization is positive. The time profile of pos. 4 (Fig. 4(e)) shows two peaks at 02:55 and 02:57 UT and its polarization in negative (opposite to that of pos. 3). Obviously pos. 3 and pos. 4 represent two oppositely polarized footpoints of this flaring loop. Also the pos. 4 time profile has a secondary peak at $\sim 03: 05$ UT, which corresponds closely to the BATSE secondary peak in the energy range 25-300 keV. Clearly these energetic electrons are produced primarily at one footpoint (pos. 4) of the first flaring loop, with possible contribution from another footpoint (pos. 2) of the other flaring loop.

Photon spectral indices measured from the microwave emission at 17 and $34 \mathrm{GHz}$ for the positions 1 and 2 near the main peak are -0.67 and -1.84 respectively.

Figure 5 shows a set of total intensity images at 17 and $34 \mathrm{GHz}$ along with the available SXT images (from $\sim 03: 19$ to $\sim 04: 16 \mathrm{UT}$ ) at selected times. Also shown in Fig. 5 is an overlay of $17 \mathrm{GHz}$ contours over the corresponding SXT image at 03:19 UT. The brightness temperature of the brightest (southern) microwave source at $17 \mathrm{GHz}$ was about 300 thousand Kelvin at 02:52:41 UT, during one of spikes in the rise phase. Its immediate vicinity had brightness temperature of only 25-35 thousand Kelvin. Similar relation was found during the next spike at 02:53:51, about 900 thousand Kelvin in the brightest blob as opposed to 50-100 thousand Kelvin in the vicinity. Later the ratio of brightness temperatures between the brightest microwave source and its brightest neighborhood decreased.

Note that the initial part of the event including the main impulsive peak was not observed by Yohkoh/SXT and HXT because of Yohkoh night time. However, after it came back from night, Yohkoh was able to observe the event during the late decay phase from 03:19 UT. As a result, we were able to compare radio imaging data with the Yohkoh/SXT data only in the late decay phase. The most recent resurgence in the search for observational evidence for magnetic reconnection in solar flares used Yohkoh X-ray data primarily in the decay phase of some LDE flares. Since radio evidence is practically non-existent in this regard, we thought it would be useful to compare radio and X-ray data at least in the late decay phase.

The set of images in Fig. 5 shows several features: 1) The bright intersection point or the loop-top microwave source shows a movement of order $10 \mathrm{~km} \mathrm{sec}^{-1}$;2) One can see a great deal of similarity between the $17 \mathrm{GHz} I$-map and the SXT image. In particular, the brightest microwave sourceblob at a loop top or near the intersection of two dominant flaring loops matches rather well with the brightest feature at a loop top in the set of SXR emitting loops in the late phase of the event. 3) The combination of $I$ - and $V$-maps at 


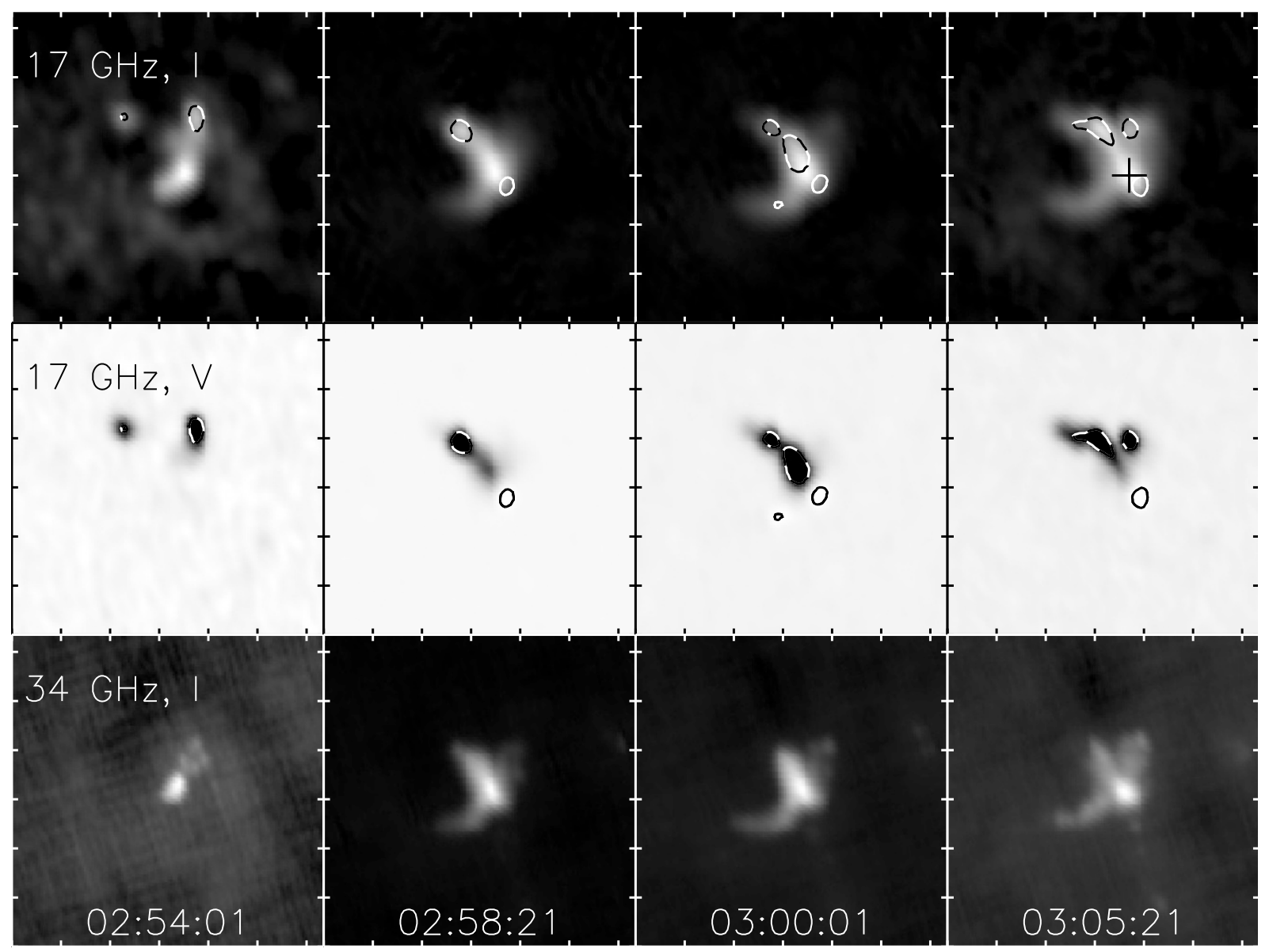

Fig. 3. The $I$ - and $V$-images at selected times (as indicated in Fig. 2 second panel) at 17 and 34 GHz. Also plotted are the $V$-contours at $17 \mathrm{GHz}$ (solid - positive polarity and dashed-negative polarity) superposed on the images in the first and second panels. The cross in the fourth image of the first panel denotes the intersection point, as discussed in the text. Field of view is $157^{\prime \prime} \times 157^{\prime \prime}$. $T_{B \text { max }}$ for each image (from left to right): $17 \mathrm{GHz}, I-0.54,10,8.1,1.8 \mathrm{MK} ; 34 \mathrm{GHz}, I-0.12,2.3,1.7,0.55 \mathrm{MK} ; 17 \mathrm{GHz}, V$ (for both polarities $)-(-190,+7),(-2800,+120),(-890,+66),(-410,+34)$ thousand Kelvin.

Kelvin in the brightest blob as opposed to 50-100 thousand Kelvin in the vicinity. Later the ratio of brightness temperatures between the brightest microwave source and its brightest neighborhood decreased.

Note that the initial part of the event including the main impulsive peak was not observed by Yohkoh/SXT and HXT because of Yohkoh night time. However, after it came back from night, Yohkoh was able to observe the event during the late decay phase from 03:19 UT. As a result, we were able to compare radio imaging data with the Yohkoh/SXT data only in the late decay phase. The most recent resurgence in the search for observational evidence for magnetic reconnection in solar flares used Yohkoh X-ray data primarily in the decay phase of some LDE flares. Since radio evidence is practically nonexistent in this regard, we thought it would be useful to compare radio and X-ray data at least in the late decay phase.

The set of images in Fig. 5 shows several features: 1) The bright intersection point or the loop-top microwave source shows a movement of order $10 \mathrm{~km} \mathrm{sec}^{-1}$; 2) One can see a great deal of similarity between the $17 \mathrm{GHz} I$-map and the SXT image. In particular, the brightest microwave source-blob at a loop top or near the intersection of two dominant flaring loops matches rather well with the brightest feature at a loop top in the set of SXR emitting loops in the late phase of the event. 3) The combination of $I$ - and $V$-maps at both 17 and $34 \mathrm{GHz}$ suggests to us that the bright microwave source-blob is not at the footpoint of a flaring loop. 4) From the relation between brightness temperatures of the brightest loop-top blob and nearby arches we conclude that the enhanced brightness of the blob is not a consequence of the superposed brightness of two intersecting loops. 5) In general, there is good correspondence between the microwave images and the SXT images in the late phase of the event, suggesting that microwave emission probably results from the SXR emitting thermal plasma.

\section{Discussion and Concluding Remarks}

Several phases can be distinguished in the course of the flare according to microwave time profiles: (1) an initial phase, when an impulsive peak was recorded at $17 \mathrm{GHz}$ at $\sim 02: 53 \mathrm{UT} ;(2)$ the main impulsive phase, when we observe the impulsive gyrosynchrotron emission from the footpoint region and broader impulsive emission from the loop-top region where we seem to 


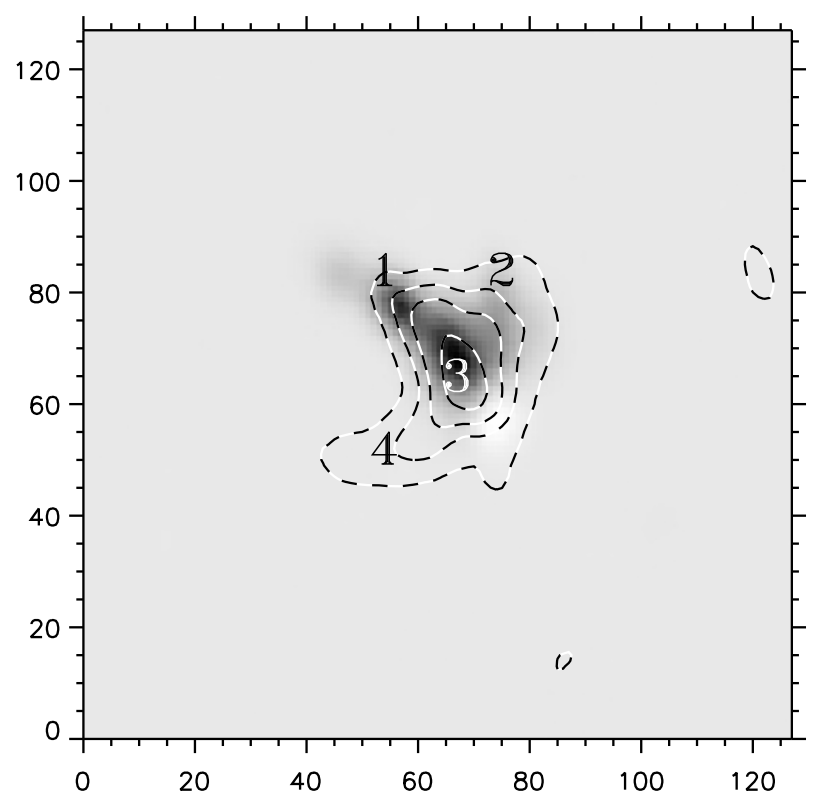

a
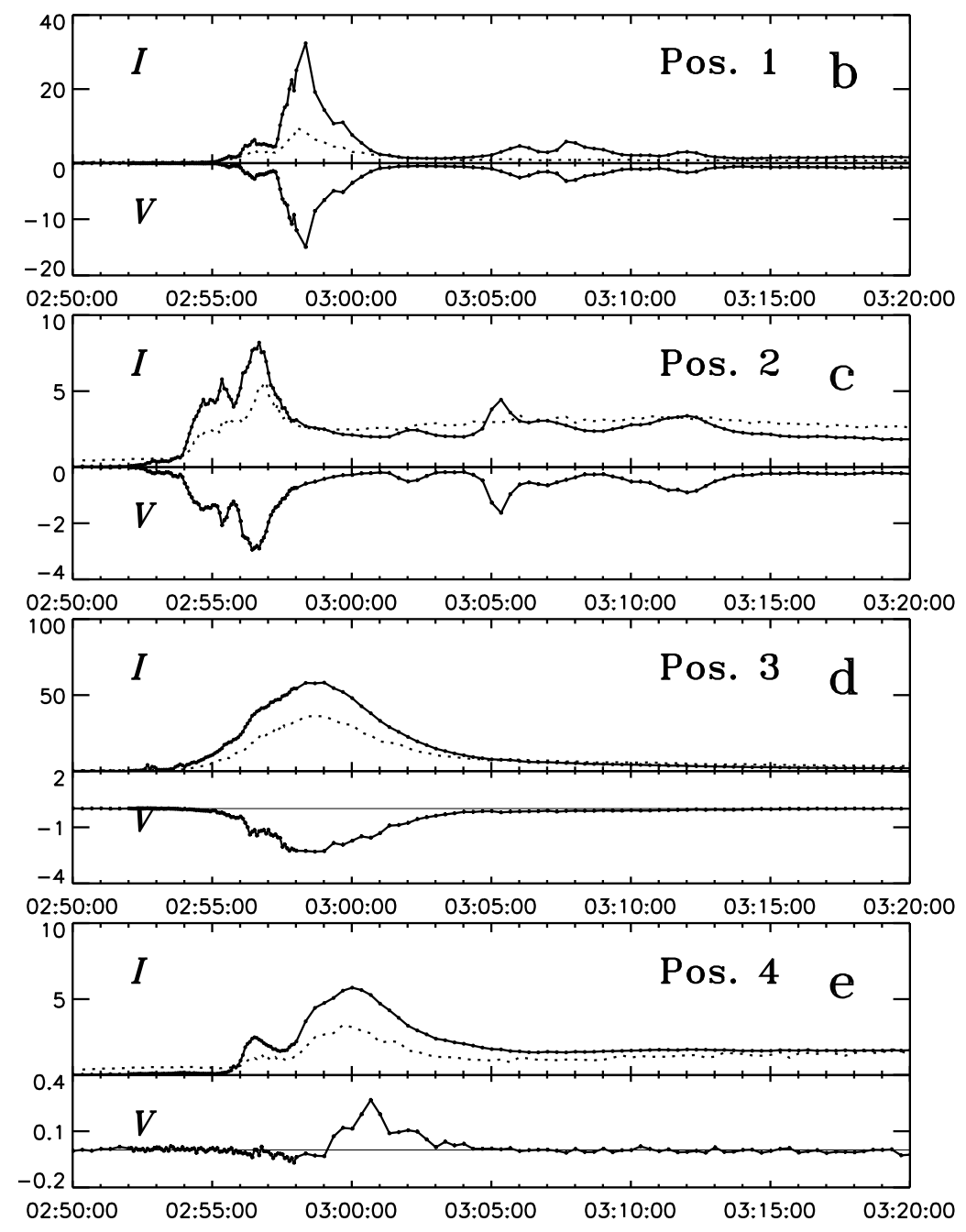

Fig. 4. Top: (a): $17 \mathrm{GHz}$ polarization map (Stokes $V$ ) of the flaring structure, averaged over the period 02:55 to 03:05 UT is shown as grey scale background; superposed on it are the $17 \mathrm{GHz}$ contours of total intensity (Stokes $I$ ), averaged over the same period. Contour levels are $(0.02,0.075,0.2,0.5)$ of $T_{B \max }=5.8 \mathrm{MK}$. Field of view is $157^{\prime \prime} \times 157^{\prime \prime}$. Bottom: (b-e) show microwave time profiles (s.f.u.) for some positions of the flaring structure which are marked in the image (a). Total flux is taken over the area $\sim 20^{\prime \prime} \times 20^{\prime \prime}$. In the upper panels of each pair of plots the total intensity at $17 \mathrm{GHz}$ is shown by solid lines, and the total intensity at $34 \mathrm{GHz}$ is shown by dotted lines. Polarization (Stokes $V$ component) is plotted in lower panels. Zero level is shown by thin lines. 


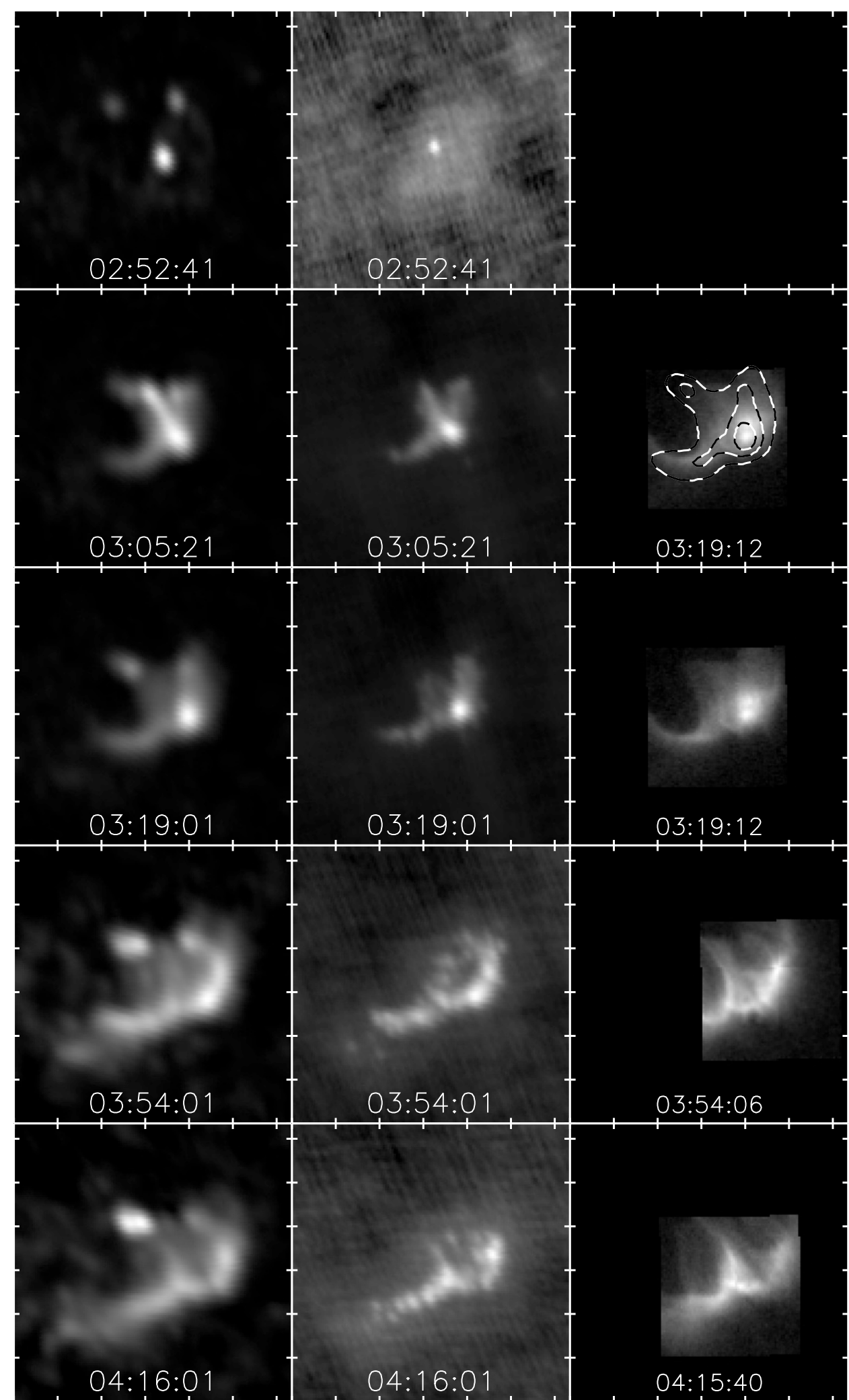

Fig. 5. A sequence of total intensity images at 17 and $34 \mathrm{GHz}$ (first two columns) at selected times (see Fig. 2) along with available SXT images from 03:19 to about 04:16 UT (third column). The microwave images at 17 and $34 \mathrm{GHz}$ are shown to portray the slow motion of the bright microwave blob as a function of time. Also shown is the overlay of the microwave source at 03:19:12 UT on the corresponding SXT image at the same time (second row, third column). Field of view is $157^{\prime \prime} \times 157^{\prime \prime}$. 
from a footpoint of one flaring loop (see pos. 4 time profile); another footpoint of the other flaring loop may have some contribution also (see pos. 2 time profile). Prolonged acceleration processes in the late decay phase of an LDE flare was also observed by Altyntsev et al. (1999).

The impulsive peak that occurred in the initial phase at 02:53 UT (see Fig. 2 bottom panel) originated from a region close to the point of intersection of the microwave flaring loops involved. From temperature light curve derived from GOES data (see Fig. 1(a)), we know that an impulsive heating occurred at about 02:52-02:54 UT. From CGRO/BATSE records, we also know that electrons with energies of up to at least $100 \mathrm{keV}$ contributed to HXR emission during this peak. Electrons with energies of $\gtrsim 300 \mathrm{keV}$ contributed to the main phase emission at 02:58 UT and at 03:05 UT. Fine time structures recorded by NoRH support the view that nonthermal processes occurred in the initial phase in this region.

We have shown that non-thermal emissions in both early phase (02:52-02:54 UT) and in the main phase occur in the apparent intersection region. The late phase thermal emission also occurs in the same region as evidenced by the close correspondence in spatial structure between $17 \mathrm{GHz}$ and SXT images at 03:19 UT (after Yohkoh came back from night time). This correspondence is shown at 03:19 UT (see Fig. 5). So both thermal and non-thermal flaring processes occurred in this region, and we suggest the possibility that this bright loop-top source, which seems to be located near the intersection of two flaring loops in microwaves, may be a site of energy release via magnetic reconnection process. The fact that the bright intersection point or loop-top microwave source shows a movement of order $\sim 10 \mathrm{~km} \mathrm{~s}^{-1}$ (see Fig. 5), similar to what Tsuneta et al. (1992) observed in X-rays in the case of the 1992 February 21 LDE flare (Yohkoh/SXT data), and which Tsuneta et al. considered to be a consequence of reconnection, adds strength to our plausibility argument for possible evidence of reconnection in the present radio case. One expects a speed of this order from the rising motion of the inner X-point of reconnection (Tsuneta et al., 1992). Thus the bright microwave blob is not entirely a region of emission from trapped electrons in the main phase or bremsstrahlung in the decay phase (which we see in SXR in the late phase of the flare).

The reason we believe that we are dealing with a possible signature of magnetic reconnection is that the flaring structure seems to consist of at least two apparently intersecting flaring loops and at the intersection point or slightly above it, there is a bright blob, resembling the HXR loop-top source, sometimes observed in flares (see Masuda et al., 1994). So, two scenarios are possible: bright microwave emission at the intersection of two seemingly intersecting flaring loops or a loop-top compact bright source like the HXR loop-top source. Both scenarios are consistent with the possibility of reconnection occurring at the apparent intersection region.

The flare configuration as described here is not entirely consistent with the "CSHKP" standard model, because some features do not seem to be compatible with this model. For example, the intersection region seems to be the location of both thermal and non-thermal processes as exhibited by impulsive fine structure elements in the rise phase; non-thermal microwave sources are associated with two brightest loops and are compact unlike widely distributed $\mathrm{H}_{\alpha}$ ribbons, suggesting that acceleration processes involved some kind of interaction between these loops; the time profiles of the looptop and footpoint sources, although they peak at the same time, are not identical.

Acknowledgments. We wish to thank Dr. H. Hudson for making some useful comments on the paper. This research was done during a tenure of the two authors as visiting scientists at NAOJ and NRO. The work at Maryland was supported by NSF grants ATM 99-09809 and INT 98-19917, and NASA grants NAG 5-8192 and NAG 57370. The work in Irkutsk was partly supported by Russian RFFI grants 00-02-16819, 99-07-90038, 00-15-96710, and the program "Astronomy". The authors wish to thank the staff of NAOJ and NRO for their help and hospitality during their stay at the observatories.

\section{References}

Altyntsev, A. T., V. V. Grechnev, H. Nakajima, K. Fujiki, M. Nishio, and D. V. Prosovetsky, The limb flare of November 2, 1992: Physical conditions and scenario, Astron. Astrophys. Suppl. Ser., 135, 415-427, 1999.

Antonucci, E., C. Benna, and B. Somov, Interpretation of the observed plasma turbulent velocities as a result of magnetic reconnection in solar flares, Astrophys. J., 456, 833-839, 1996.

Gold, T. and F. Hoyle, On the origin of solar flares, MNRAS, 120, 89-105, 1960.

Holman, G. H., M. R. Kundu, and K. Papadopoulos, Electron pitch angle scattering and the impulsive phase microwave and hard X-ray emission from solar flares, Astrophys. J., 257, 354-360, 1982.

Kundu, M. R., Observational evidence for magnetic reconnection in microwave solar bursts, in Unstable Current Systems and Plasma Instabilities in Astrophysics, IAU Symp. No. 107, edited by M. R. Kundu and G. Holman, pp. 185-190, 1985.

Kundu, M. R., E. J. Schmahl, T. Velusamy, and L. Vlahos, Radio imaging of solar flares using the very large array: New insights into flare process, Astron. Astrophys., 108, 188-194, 1982.

Lee, J. W. and D. E. Gary, Microwave bursts and electron pitch angle, in High Energy Solar Physics: Anticipating HESSI, edited by Ramaty and Mandzhavidze, ASP conf. Series., 206, pp. 323-328, 2000.

Masuda, S. et al., A loop-top hard X-ray source in a compact solar flare as evidence for magnetic reconnection, Nature, 371, 495-497, 1994.

McKenzie, D. and H. S. Hudson, X-ray observations of motions and structure above a solar flare arcade, Astrophys. J. Lett., 519, L93-L96, 1999.

Parker, E. N., Sweet's mechanism for merging magnetic fields in conducting fluids, J. Geophys. Res., 62, 509-520, 1957.

Petschek, H. E., Magnetic field annihilation, in Proc. AAS-NASA Symp. on Physics of Solar Flares, edited by W. N. Hess, pp. 425-439, NASA SP-50, Washington, D.C., 1964.

Shibata, K., Coronal X-ray jets observed with Yohkoh/SXT, Adv. Space Res., 17(4/5), 197-200, 1996.

Shibata, K. et al., Hot plasma ejections associated with compact-loop solar flares, Astrophys. J. Lett., 451, L83-L85, 1995.

Sweet, P. A., The neutral point theory of solar flares, in Electromagnetic Phenomena in Cosmical Physics, IAU Symp. 6, edited by B. Lehnert, pp. 123-134, 1958.

Tsuneta, S., Structure and dynamics of magnetic reconnection in a solar flare, Astrophys. J., 456, 840-849, 1996.

Tsuneta, S. et al., Observation of a solar flare at the limb with the Yohkoh soft X-ray telescope, PASJ, 44, L63-L69, 1992.

M. R. Kundu (e-mail: kundu@astro.umd.edu) and V. V. Grechnev 